\title{
AN AGENT-BASED SIMULATION FRAMEWORK TO ANALYZE THE PREVALENCE OF CHILD OBESITY
}

\author{
Adrian Ramirez-Nafarrate ${ }^{1}$ \\ J. Octavio Gutierrez-Garcia ${ }^{2}$ \\ ${ }^{1}$ Department of Industrial and Operations Engineering \\ ${ }^{2}$ Department of Computer Science \\ Instituto Tecnologico Autonomo de Mexico \\ Mexico, DF 01080, MEXICO
}

\begin{abstract}
Child obesity is a public health problem that is of concern of several countries around the world. Long-term effects of child obesity include prevalence of chronic diseases, such as diabetes and heart-related illnesses. This paper presents an agent-based simulation framework to analyze the evolution of obesity in school-age children. In particular, in this paper we evaluate the impact of physical activity on the prevalence of child obesity using an agent-based simulation model. Simulation results suggest that the fraction of overweight and obese children at the end of elementary school can be reduced by doing physical activity with moderate intensity.
\end{abstract}

\section{INTRODUCTION}

Child obesity is a public health problem for several countries. According to the Organisation for Economic Co-operation and Development (OECD), about $20 \%$ of children in OECD countries are affected by overweight or obesity. However, in Greece, the United States, Italy and Mexico, the percentage of overweight or obese children is above 30\% (OECD 2012). Obesity has several negative effects on the health status of children and adults. The National Institute of Health (NIH) states that obesity increases the risk of diabetes, hypertension, sleep apnea, liver disease, stroke and some types of cancer (NIH 1998). Therefore, an obese person incurs, on average, $25 \%$ higher health expenditures than a person with a healthy weight. Furthermore, in the United States, the expenditures on obesity-related problems represents between 5\% to $10 \%$ of the overall health expenditures (OECD 2012).

According to the Centers for Disease Control and Prevention (CDC), child obesity is caused mainly by excess caloric intake. Thus, obese children consume too many calories without doing enough physical activity (CDC 2013). In regard to children, most of both the caloric intake and the caloric expenditure take place in school and at home (Levy et al. 2011).

Some countries have developed plans to reduce the prevalence of obesity during childhood based on policies applied to schools. For example, Huang (2013) presents guidelines for the architecture of school buildings to promote healthy behaviors, including healthy eating principles. These guidelines were applied in the redesign of a school in Virginia. In Mexico, the government passed a new act on education in february 2013 (Presidencia de Mexico 2013). This act states that the government will promote healthy food and will prohibit the sale of junk food in elementary schools.

In order to implement changes in schools, tools to assess the decisions of policy-makers must be designed. In this paper, we present an agent-based simulation (ABS) framework that can help policy-makers to design meal menus and physical activity programs for school-age children to reduce the prevalence of obesity during childhood. In addition, we present preliminary experiments using the agent-based model to study 


\section{Ramirez-Nafarrate and Gutierrez-Garcia}

the effect of different levels of intensity of physical activity during elementary school. The remaining sections of the paper are organized as follows: in Section 2 we present the literature review relevant to this work, in Section 3 we present the proposed ABS framework and describe the experiments and results obtained, and finally, in Section 4 we present the concluding remarks and future research lines.

\section{LITERATURE REVIEW}

Obesity has been widely studied in the health literature. However, since this work focuses on ABS of child obesity, we present in this section some of the most relevant work in the modeling and simulation of obesity.

Jódar et al. (2008) present a systems dynamics model to study the evolution of child obesity. The model comprises six compartments: normal weight, latent individuals, overweight, obese, overweight on diet and obese on diet. The authors define transition rates and estimate the input parameters. The authors conclude that the consumption of food with poor nutritional characteristics contribute significantly to increase obesity.

Ma and Frick (2011) simulate a variety of intervention strategies and analyze their cost-effectiveness. The authors show that targeted interventions on obese population could yield higher cost savings than population-based interventions for children between 0 and 6 years old.

Bruzzone et al. (2012) propose an agent-based simulation model to study obesity in adults. The authors discuss the importance of collecting and analyzing the appropriate data to construct the model. Similarly, Christakis and Fowler (2007) analyze an interconnected social network using longitudinal statistical models and study the correlation of weight gain among friends and family. Their results suggest that there is a relative high probability of becoming obese if a close friend or family member is obese.

Chalkias et al. (2013) use statistical methods to analyze the relationship between child obesity and socioenvironmental status in Athens, Greece. The authors suggest that areas characterized by low educational level, high population density, low family income and small amount of green/recreational zones are highly positively correlated with the prevalence of child obesity in those areas.

A comprehensive study on simulation models applied to obesity-related problems is presented in Levy et al. (2011). The authors classify the existing models depending on their focus: health and economic outcomes, trends in obesity as a function of past trends, physiologically based behavioural models, environmental contributors to obesity and policy interventions. The authors highlight that simulation models to evaluate policy interventions are at an early stage and they require to be exploted to analyze complex problems such as obesity.

In this paper we delineate an agent-based simulation framework to study the prevalence of child obesity. In particular, we design a model that can be used by decision makers to analyze the effectiveness of intervention strategies in schools. This model contributes to the literature by presenting an agent-based model applied to child obesity that captures important elements of home and school environments, such as eating and physical activity habits. The architecture of the model is self-explanatory for a better understanding of decision makers, as suggested by Levy et al. (2011). We present a preliminary experiment and its results with real data and we emphasize significant differences between children and adults for the computation of body mass index, and caloric intakes and expenditures.

\section{MODELING FRAMEWORK AND EXPERIMENTS}

In this section we describe the proposed modeling framework based on ABS to study the prevalence of child obesity. We use ABS models because they allow to analyze a complex system with autonomous agents (Macal and North 2009).

\subsection{Agent-Based Simulation Model}

In the proposed ABS framework, agents are the children to analyze. We assume that children have eight attributes: age, gender, weight, height, body mass index (BMI), weight status category (underweight, 


\section{Ramirez-Nafarrate and Gutierrez-Garcia}

healthy weight, overweight or obese), daily caloric intake, and energy expenditure. The first four attributes are independent, the BMI and the weight status category are computed based on these values. Caloric intake and energy expenditure are random variables. Each of these attributes are initialized at the beginning of the simulation run. The initial values or distribution for each attribute must be defined according to the population to be analyzed. Thus, if the model is going to be used to evaluate intervention strategies at elementary schools, then the children must be defined with attribute values according to children with age between 6 and 12 years old. Figure 1 presents a scheme of agent's attributes.

\section{Child attributes}

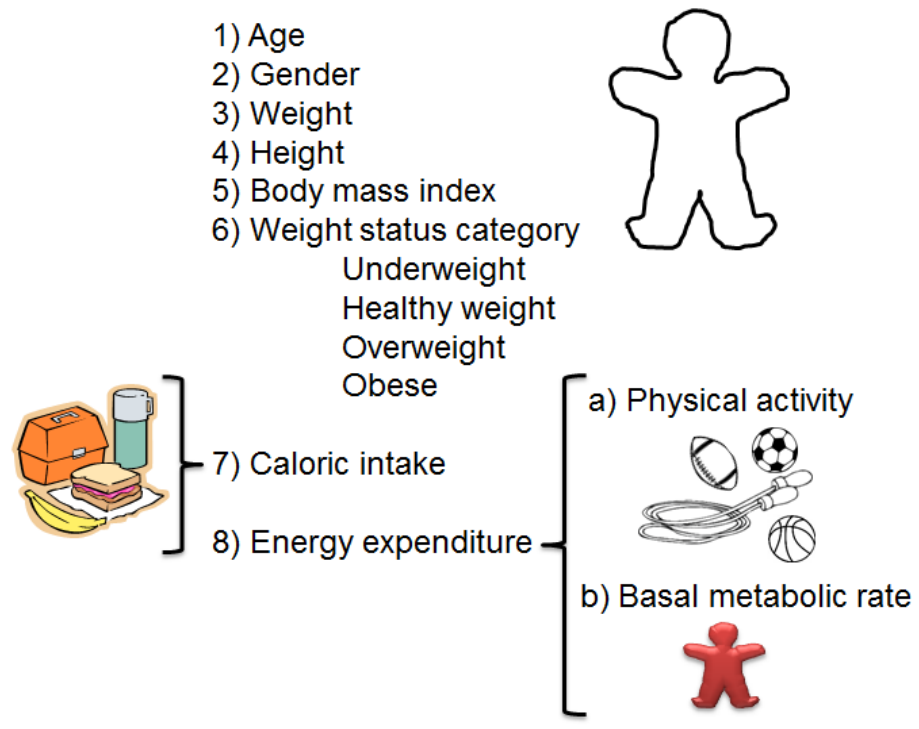

Figure 1: Attributes of agents

Once that initial values for the attributes of each child have been defined, then the simulation run can be executed for some amount of simulation time. In the proposed framework, we assume that a simulation is run for a given number of days. At each simulated day, the caloric intake and the energy expenditure of each child is obtained based on probability distributions, child profile (eating and exercise habits) and/or food and physical activity policies at school. Based on analysts assumptions of caloric intake and energy expenditure, the height and weight of each child is updated and the BMI of each child is computed. The $\mathrm{BMI}$ is a measure of weight in relation to height that is widely used to determine the weight status category of individuals, including children (Eknoyan 2008). The weight status category of each child is updated based on his/her BMI value. During the simulation run, the required statistics are collected (i.e. percentage of children on each weight status category). Figure 2 shows the simulation algorithm.

\subsection{Input Data and Experimentation}

In order to run experiments for analyzing child obesity, it is necessary to set up appropiate values for input data and updating functions because there are significant differences between children and adults in several aspects, such as energy expenditure and weight status category.

The weight status classification for children depends on gender and age. The CDC have guidelines and tables to classify children in underweight, healthy weight, overweight or obese. The CDC guidelines are based on BMI percentiles, which allow one to compare the relative position of a child's BMI among children of the same age and gender (CDC 2011). Table 1 presents how to determine the weight status of children based on these guidelines. 


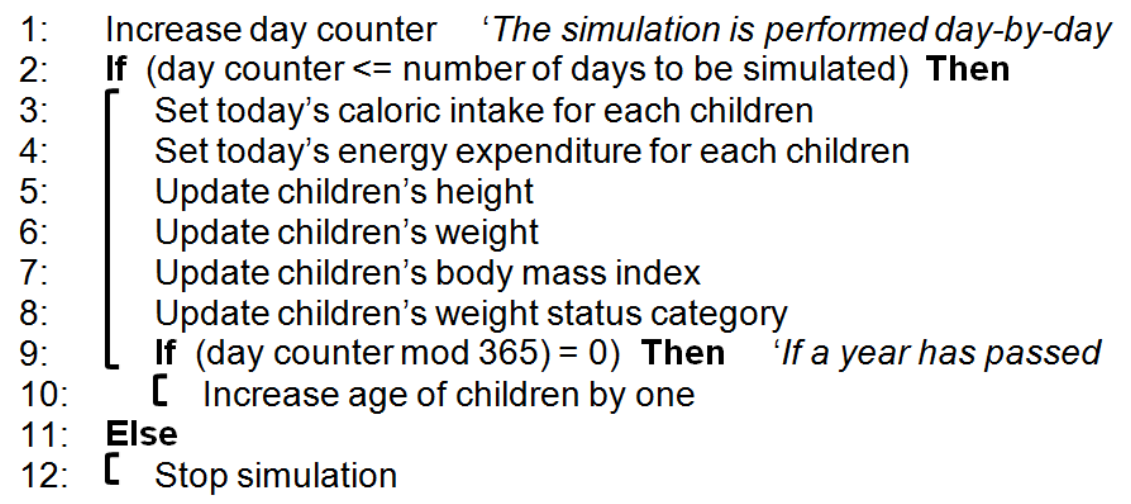

Figure 2: ABS algorithm to study child obesity

Table 1: Determination of weight status category for children

\begin{tabular}{cc} 
Weight status category & Percentile range \\
\hline Underweight & Less than the 5th percentile \\
Healthy weight & 5th percentile to less than the 85th percentile \\
Overweight & 85th to less than the 95th percentile \\
Obese & Equal to or greater than the 95th percentile
\end{tabular}

The BMI values for these percentiles change as the age of the child increases. In order to implement the classification of weight status in the ABS model, functions for the 5th, 85th and 95th percentiles published by the CDC (CDC 2010) were fitted using regression, where the independent variable is the child's age in months. Figure 3 shows the regression functions for the 5th and 85th percentiles for boys between 6 and 12 years old. Hence, if the BMI of a 10 year-old boy is 19.5, then the boy is classified as overweight.

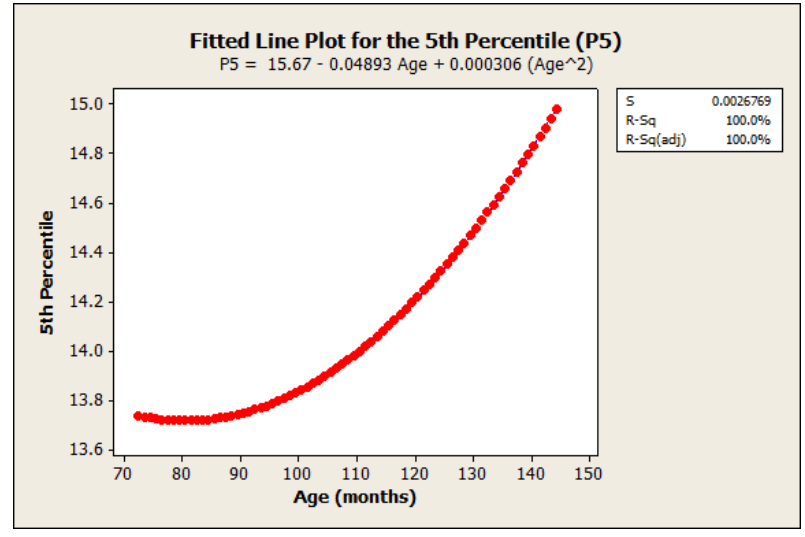

(a) BMI for the 5th percentile

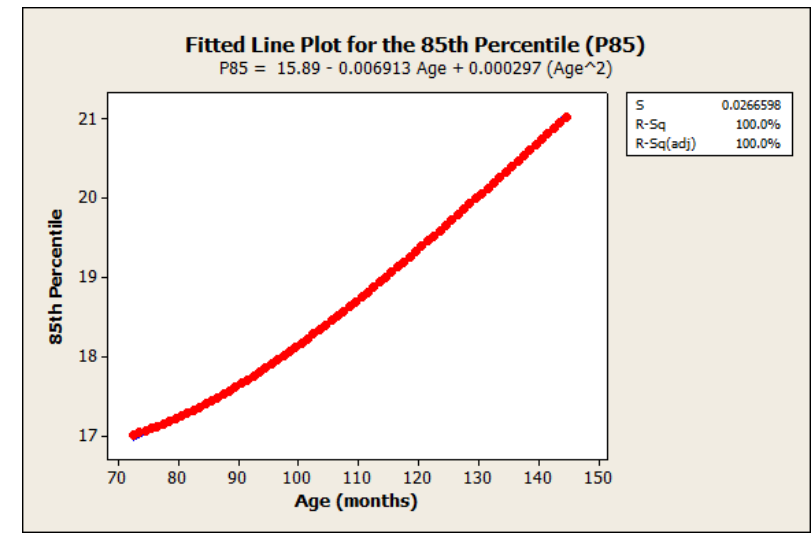

(b) BMI for the 85th percentile

Figure 3: Regression functions for the 5th and 85th BMI percentiles for boys

In order to determine the daily net difference between caloric intake and expenditure of a child, we defined a caloric balance equation of the same form that the one presented by Cutler et al. (2003). The caloric balance equation is given by Equation (1) and it is based on caloric intake and energy expenditure of adults.

$$
K_{\text {out }}=\alpha+(\beta+E) W+0.1 K_{\text {in }}
$$




\section{Ramirez-Nafarrate and Gutierrez-Garcia}

where:

$K_{\text {out }}$ : caloric expenditure

$W$ : weight in kilograms

$K_{\text {in }}$ : caloric intake

$E$ : energy expenditure due to physical activity

$\alpha$ and $\beta$ : constants that depend on gender

Equation (1) can be interpreted as follows, there are three ways to burn calories: basal metabolism, digestion process and physical activity. The first process is related to the minimum amount of calories required to keep the body alive at rest and is given by $\alpha+\beta W$. Schofield et al. (1985) estimate the combination $(\alpha, \beta)$ as $(879,11.6)$ and $(829$ and 8.7$)$ for men and women, respectively. The second process is digestion, which is the food processing mechanism of the body. On average, the energy expenditure required by digestion is $10 \%$ of the caloric intake. The third way to burn calories is through physical activity. The average number of calories burned by kilogram through physical activity depends on the intensity of the activity and its duration.

Harrell et al. (2005) suggest that energy expenditure in children is greater than in adults. For example, the energy expenditure at rest for boys and girls between 8 and 11 years old is, on average, $71 \%$ higher than in adults. The caloric expenditure due to physical activity is also greater in children of these ages compared with adults.

In order to determine the caloric intake for each child in the model for a specific day (line 3 in Figure 2 ), we compute the minimum amount of calories required by a child $\left(K_{\min }\right)$ as shown in Equation (2), and take into account the constant values as suggested by Schofield et al. (1985). This equation includes two constants: 1.1 for digestion, and 1.71 for the energy expenditure at rest in children compared with adults.

$$
K_{\text {min }}=(\alpha+\beta W) *(1.71 * 1.1)
$$

Then, the caloric intake of a child for a specific day $\left(K_{i n}\right)$ is computed based on a probability distribution. For the experiments presented on this paper, we assume that the caloric intake is uniformly distributed between $95 \%$ and $117 \%$ of the minimum required. These values suggest that a child tends to eat more calories than the required by the body. In order to use the model to evaluate intervention policies in schools, it would be necessary to estimate $K_{i n}$ based on home and school eating habits. These estimates would vary depending on demographics and cultural characteristics. More detail could be added to the model by considering caloric properties of the food available in schools.

After estimating the caloric intake of a child for a specific day, the simulation model determines the energy expenditure per kilogram for that day $(E)$. The esimation of $E$ (line 4 in Figure 2 ) is based on the type of physical activity that a child does on a given day. Harrell et al. (2005) present tables for the energy expenditure $\left(\mathrm{kcal} / \mathrm{kg}^{*} \mathrm{hr}\right)$ in children based on the intensity of the activity. Table 2 presents examples of physical activities and their caloric expenditure. Table 2 suggests that watching one hour of TV implies burning only 1 calorie per kilogram additional to the minimum required (1.71), while running during an hour implies burning 6.29 calories per kilogram additional to the minimum required.

Table 2: Energy expenditure by physical activity in children between 8 and 11 years old presented by Harrell et al. (2005)

\begin{tabular}{ccc} 
Intensity & Activity & Energy Expenditure $(\mathrm{kcal} / \mathrm{kg} * \mathrm{hr})$ \\
\hline Sedentary & Watching TV & 1.72 \\
Sedentary & Playing video games (sitting) & 2.02 \\
Moderate & Sweeping & 4.92 \\
Moderate & Walking $(4 \mathrm{~km} / \mathrm{hr})$ & 5.22 \\
High & Walking $(5.6 \mathrm{~km} / \mathrm{hr})$ & 7.02 \\
High & Running $(8 \mathrm{~km} / \mathrm{hr})$ & 11
\end{tabular}




\section{Ramirez-Nafarrate and Gutierrez-Garcia}

In the experimentation presented in this paper, we defined three scenarios based on three levels of physical activity: low, medium and high. We assume that all the children in the simulation have the same profile for physical activity and that the amount of calories burned in a day through physical activity is uniformly distributed as shown in Table 3. The values for the distribution probabilities are based on the differences between the calories burned by the physical activity and the calories burned by the basal metabolism. For instance, if the physical activity that a child does in a day is equivalent to sweeping during one hour, that implies burning $3.2 \mathrm{kcal} / \mathrm{kg}$, which belongs to a medium level.

Table 3: Energy expenditure $(E)$ levels of physical activity for the experiments

\begin{tabular}{cc} 
Level & Energy Expenditure $(\mathrm{kcal} / \mathrm{kg})$ \\
\hline Low & $\mathrm{U}(0.5,3.5)$ \\
Medium & $\mathrm{U}(1.5,4.5)$ \\
High & $\mathrm{U}(2.5,5.5)$
\end{tabular}

For the general framework, we envisioned that the evaluation of intervention policies should distinguish between physical activity at home and physical activity at school. Therefore, physical activity plans could be designed for implementation at school.

For our experiments, we assume that the height of each child (line 5 of Figure 2) is updated each day $t$ based on a linear function of the form $H_{t}=H_{t-1}+\rho$, where $\rho$ is the average increase in height, in centimeters, in a day. In the general framework, the updating function can be adapted to any type of function that may include stochasticity.

After updating the height, the weight of each child is updated (line 6 of Figure 2) each day according to Equation (3). In this equation $H_{t}$ is the height, in centimeters, of the child at day $t$ and $\gamma$ is the average weight gain (in kilograms) due to increasing one centimeter in height. Cutler et al. (2003) estimates that, for a typical person, an increase in calorie consumption of 3500 calories increases the weight by one pound. We made a linear conversion of this factor to imply that consuming 7716 calories increases the weight by one kilogram.

$$
W_{t}=W_{t-1}+\left(H_{t}-H_{t-1}\right) \gamma+\left(\left(K_{\text {in }}-K_{\text {out }}\right) / 7716\right)
$$

The BMI for each child at day $t$ is computed using Equation (4) (line 7 of Figure 2). Then, the weight status category of each child is classified based on the BMI value for each child and the guidelines and functions as shown in Table 1 and Figure 3, respectively (line 8 of Figure 2).

$$
B M I_{t}=W_{t} / H_{t}^{2}
$$

As estimates for $\rho$ and $\gamma$, we averaged the changes in height and weight of the 50th percentile of the growth chart published by the CDC (CDC 2010). Hence, $\rho$ is assumed to be 0.015 and 0.016 for boys and girls, respectively; and $\gamma$ is assumed to be 0.6 for both, boys and girls.

In order to test the ABS framework with the estimated parameters, we set the objective of analyzing the prevalence of child obesity for a class of students of elementary school. We constructed the ABS model using Netlogo 5.0.4 (Northwestern University 2013). For each scenario in the experimentation, we considered ten replications of six years of simulation each. We chose six years because that is the duration of elementary school in Mexico. The initial population comprised 2006 -years-old children, 50\% of which were boys and the rest girls. The initial age was set to 6 years old because it is the required age to enroll to elementary schools in Mexico.

The height and weight of the initial population was generated assuming normality. In all cases, Kolmogorov-Smirnov goodness-of-fit tests confirmed a good fit with a 95\% confidence level based on the height and weight percentiles of 6-year-old children (CDC 2010). Hence, the mean and standard deviation for the height and weight of the initial population of boys are assumed to be $\mathrm{N}(115.66,5)$ and $\mathrm{N}(21,5)$, respectively; and the height and weight of the initial population of girls are assumed to be $\mathrm{N}(115.01,5)$ and $\mathrm{N}(20,5)$, respectively. 


\section{Ramirez-Nafarrate and Gutierrez-Garcia}

\subsection{Results}

The ABS model allows tracking the weight status category of children over time. For example, Figure 4 shows the fraction of boys in each weight status category over the simulation time for the three levels of physical activity. These figures suggest that the fraction of children with healthy weight increases significantly as they increase the intensity of their physical activity. However, there is a risk of becoming underweight if the intensity of the daily exercise is high. In addition, Figure 4 shows that approximately after 10 years old, the boys tend to mantain a healthy weight, probably influenced by the growth peak, which usually occur during puberty (Abbassi 1998).

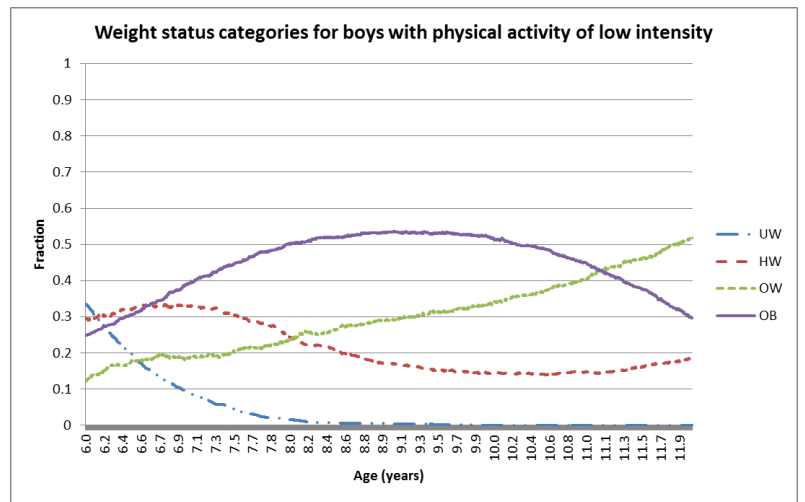

(a) Low intensity

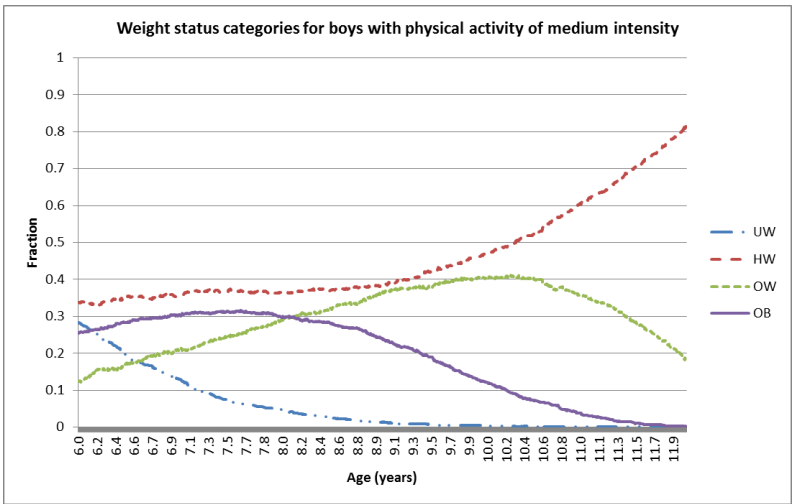

(b) Medium intensity

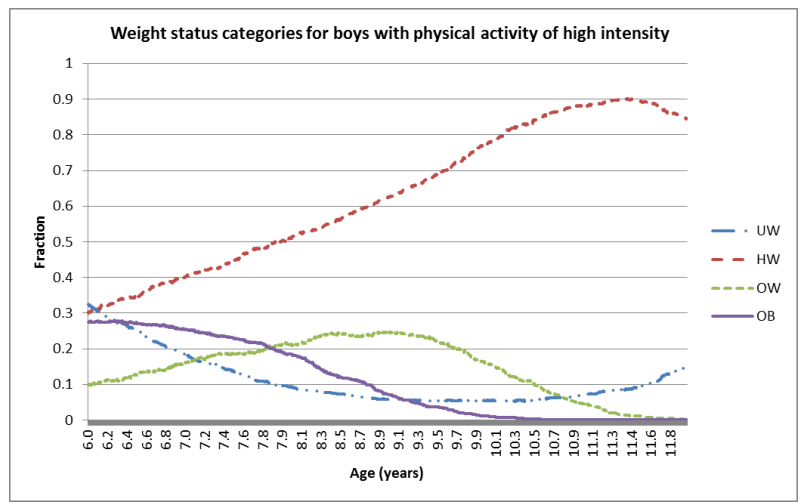

(c) High intensity

Figure 4: Weight status categories of boys for different intensity levels of physical activity (UW: underweight, HW: healthy weight, OW: overweight, OB: obese)

Figure 5(a) shows box plots for the minimum, average and maximum weight at the end of the simulation for boys for the three levels of intensity of physical activity. The weight of the children when they are 12 years old decreases as the intensity of the daily exercise increases. According to the growth tables of the CDC (CDC 2010), the median for the weight of 12-years-old boys is $40.67 \mathrm{~kg}$, which is close to the average weight of children doing physical activity of medium level: $43.4 \mathrm{~kg}$, which represents a difference of $6.74 \%$. Figure 5(b) shows box plots for the average BMI for boys at the end of the simulation. This figure suggests that doing only physical activity of low intensity produces an average BMI that is peculiar of overweight children, while the average BMI obtained by doing medium and high intensity activities is peculiar of children with healthy weight. 


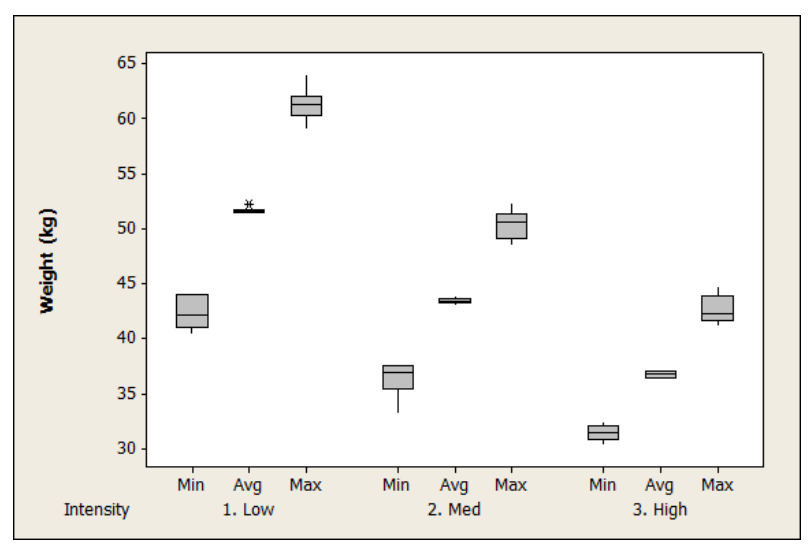

(a) Box plots for weight

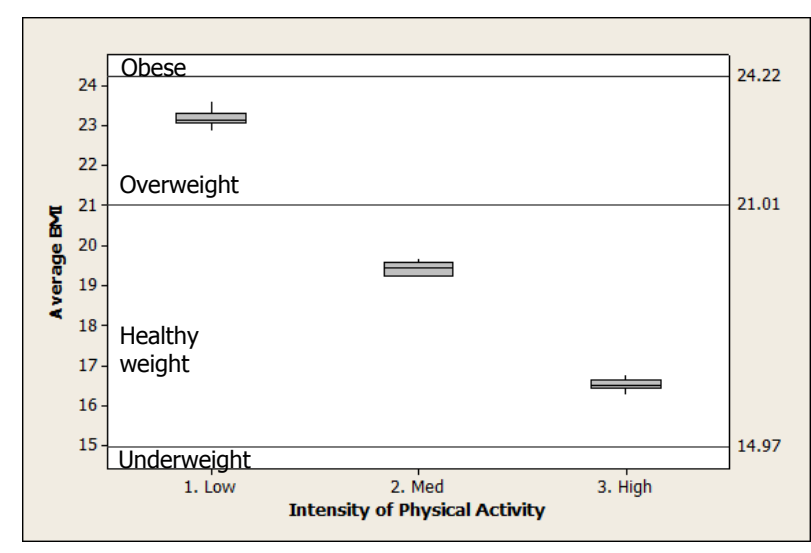

(b) Box plots for BMI

Figure 5: Box plots for weight and average BMI of boys at the end of the simulation

Table 4 presents $95 \%$ confidence intervals for the percentage of children in each category. According to the growth tables of the CDC (CDC 2010), at age of 12, 5\% of children are expected to be underweight, $80 \%$ are expected to have healthy weight, and $15 \%$ are expected to be overweight or obese. Therefore, we believe that the values defined for the medium-intensity physical activity are close to the intensity recommended to keep a healthy children population, even if they tend to consume more calories than the minimum required, as assumed in this paper. However, the input data used in this paper seem to overestimate the final weight because the final percentage of underweight children doing medium-level physical activity is smaller than the expected..

Based on this information, we also infer that children in countries like Greece, the United States, Italy and Mexico do not do sufficient exercise to burn the additional calories consumed every day. It is very likely that the average level of daily exercise of children in these countries is somewhere between the low and medium levels defined on this paper; or in other words, the fraction of children that do only sedentary activities is relatively high.

Table 4: 95\% confidence intervals on the percentage of boys in each weight status category at the end of the simulation for each intensity level of physical activity

\begin{tabular}{cccc} 
Category & Low & Medium & High \\
\hline Underweight & $(0,0)$ & $(0,0.32)$ & $(12,18)$ \\
Healthy weight & $(16,20)$ & $(79,84)$ & $(81,87)$ \\
Overweight or Obese & $(79,83)$ & $(15,21)$ & $(0,0.50)$
\end{tabular}

Similar conclusions were drawn about the effect of exercise on the weight status of girls. However, they tend to be slightly taller and have higher percentages for the healthy weight status than boys. This might be due to the fact that the growth peak in girls occurs earlier than in boys (Abbassi 1998).

\section{CONCLUSIONS}

This paper presents an ABS framework to study the prevalence of child obesity. There are many active research efforts tackling obesity-related issues, but most of them are focused on adults. Therefore, this paper contributes to existing literature by presenting an agent-based model to analyze child obesity that takes into account a great variety of aspects, such as biological functions of the body, as well as eating and exercise habits. 


\section{Ramirez-Nafarrate and Gutierrez-Garcia}

There is little quantitative information about the behavior and habits of children; however, the input data used in the experiments of this paper can be used as a starting point for a more comprehensive analysis. The experiments suggests that being consistent with physical activity of medium activity can help to reduce obesity prevalence.

However, it is important to highlight that there is not a generic case of child obesity. Consequently, the input data must be adapted to demographical, economical and cultural characteristics of the region of interest. The lack of data regarding variables related to obesity is an important problem discussed by many papers in the literature; therefore, the implementation of the proposed ABS framework requires to take samples and perform a comprehensive analysis on data before constructing the model.

We envision that the ABS framework can have useful applications, especially for school policy-makers. The following are lines of future research that are based on the proposed ABS framework:

1. Analyzing and including eating and exercise profiles for each child in the simulation model (e.g. active and passive behaviors towards physical activity).

2. Discriminating eating and exercise in school and at home.

3. Evaluating intervention strategies in schools to reduce the prevalence of child obesity. These strategies can be related to designing meal menus and physical activity programs.

4. Analyzing the effect of social relationships in the prevalence of child obesity by taking advantage of the features of ABS.

\section{ACKNOWLEDGMENTS}

This research is supported by Asociación Mexicana de Cultura A.C. In addition, the first and second authors acknowledge the support provided by CONACYT through grants no. 174548 and no. 192153, respectively.

\section{REFERENCES}

Abbassi, V. 1998. "Growth and normal puberty". Pediatrics 102 (Supplement 3): 507-511.

Bruzzone, A. G., V. Novak, and F. Madeo. 2012. "Obesity epidemics modelling by using intelligent agents". SCS M\&S Magazine (9): 18-24.

CDC 2010. "CDC Growth Charts". Accessed May. 18, 2013. http://www.cdc.gov/growthcharts/cdc_charts. htm.

CDC 2011. "About BMI for children and teens". Accessed May. 18, 2013. http://www.cdc.gov/healthyweight/ assessing/bmi/childrens_bmi/about_childrens_bmi.html.

CDC 2013. "Overweight and Obesity". Accessed May. 18, 2013. http://www.cdc.gov/obesity/childhood/ problem.html.

Chalkias, C., A. G. Papadopoulos, K. Kalogeropoulos, K. Tambalis, G. Psarra, and L. Sidossis. 2013. "Geographical heterogeneity of the relationship between childhood obesity and socio-environmental status: Empirical evidence from Athens, Greece". Applied Geography 37:34-43.

Christakis, N. A., and J. H. Fowler. 2007. "The spread of obesity in a large social network over 32 years". New England journal of medicine 357 (4): 370-379.

Cutler, D., E. Glaeser, and J. Shapiro. 2003. "Why have Americans become more obese?". Technical report, National Bureau of Economic Research.

Eknoyan, G. 2008. "Adolphe Quetelet (1796-1874)the average man and indices of obesity". Nephrology Dialysis Transplantation 23 (1): 47-51.

Harrell, J. S., R. G. McMurray, C. D. Baggett, M. L. Pennell, P. F. Pearce, S. I. Bangdiwala et al. 2005. "Energy costs of physical activities in children and adolescents". Med Sci Sports Exerc 37 (2): 329-36.

Huang, T. T. 2013. "Healthy Eating Design Guidelines for School Architecture". Preventing chronic disease 10.

Jódar, L., F. J. Santonja, and G. González-Parra. 2008. "Modeling dynamics of infant obesity in the region of Valencia, Spain". Computers \& Mathematics with Applications 56 (3): 679-689. 


\section{Ramirez-Nafarrate and Gutierrez-Garcia}

Levy, D., P. Mabry, Y. Wang, S. Gortmaker, T.-K. Huang, T. Marsh, M. Moodie, and B. Swinburn. 2011. "Simulation models of obesity: a review of the literature and implications for research and policy". Obesity reviews 12 (5): 378-394.

Ma, S., and K. D. Frick. 2011. "A simulation of affordability and effectiveness of childhood obesity interventions". Academic Pediatrics 11 (4): 342-350.

Macal, C. M., and M. J. North. 2009. "Agent-based modeling and simulation”. In Proceedings of the 2009 Winter Simulation Conference, edited by B. J. A. D. M. D. Rossetti, R. R. Hill and R. G. Ingalls, 86-98. Piscataway, New Jersey: Institute of Electrical and Electronics Engineers, Inc.

NIH 1998. "Clincal Guidelines on the Identification, Evaluation, and Treatment of Overweight and Obesity in Adults". Accessed May. 18, 2013. http://www.nhlbi.nih.gov/guidelines/obesity/ob_gdlns.pdf.

Northwestern University 2013. "Netlogo". Accessed May. 18, 2013. http://ccl.northwestern.edu/netlogo/.

OECD 2012. "OECD Obesity Update 2012". Accessed May. 18, 2013. http://www.oecd.org/health/ 49716427.pdf.

Presidencia de Mexico 2013. "Reforma Educativa para Transformar a Mexico". Accessed May. 18, 2013. http://www.presidencia.gob.mx/wp-content/uploads/2012/12/Reforma-Educativa.pdf.

Schofield, W., C. Schofield, and W. P. T. James. 1985. Basal metabolic rate: review and prediction, together with an annotated bibliography of source material. J. Libbey.

\section{AUTHOR BIOGRAPHIES}

ADRIAN RAMIREZ-NAFARRATE is an Associate Professor in the Department of Industrial \& Operations Engineering at the Instituto Tecnologico Autonomo de Mexico. His research interests include modeling, simulation and analysis of healthcare delivery systems. He received a Ph.D. degree in Industrial Engineering from Arizona State University, an MS in Manufacturing Systems from ITESM and a BS in Industrial Engineering from Universidad de Sonora. His email address is adrian.ramirez@itam.mx.

J. OCTAVIO GUTIERREZ-GARCIA is an Associate Professor in the Department of Computer Science at Instituto Tecnologico Autonomo de Mexico. His current research interests include Cloud computing, distributed artificial intelligence, and agent-based modeling. He received his Ph.D. in Electrical Engineering and Computer Science from CINVESTAV and Grenoble Institute of Technology, respectively. He has served as a reviewer for numerous international conferences and as a member of various editorial boards of scientific journals. His email address is octavio.gutierrez@itam.mx. 